\title{
A Reinforced Tabu Search Approach for 2D Strip Packing
}

\author{
Giglia Gómez-Villouta, Jean-Philippe Hamiez*, and Jin-Kao Hao \\ Université d'Angers / LERIA, 2 boulevard Lavoisier, 49045 Angers CEDEX 01, France \\ \{gomez, hamiez,hao\}@info.univ-angers.fr
}

\begin{abstract}
This paper deals with a particular "packing" problem, namely the two dimensional strip packing problem, where a finite set of objects have to be located in a strip of fixed width and infinite height. The variant studied here considers regular items (they are rectangular to be more precise) that must be packed without overlap, rotations being not allowed. The objective is to minimize the height of the resulting packing. For this problem, we present a local search algorithm based on the well-known tabu search method. While tabu search is sometimes said to be a generic search engine (or "metaheuristic"), we reinforce two important components of our tabu search strategy to try to include problem knowledge: The fitness function incorporates a measure related to the empty spaces and diversification relies on a set of historically "frozen" objects. The resulting reinforced tabu search approach is evaluated on a set of well-known hard benchmark instances and compared with some stateof-the-art algorithms.
\end{abstract}

Keywords: Tabu search, strip packing, guided diversification

\section{INTRODUCTION}

In packing problems, "small" items (also called "boxes", "modules", "objects", or "pieces" e.g.) of various shapes (regular or not) and dimensions have to be packed (i.e. located) without overlap, with rotation and "guillotine" cuts (see Figure 1) allowed or not, in other "larger" items of regular forms or not . These larger objects are usually called "containers" or "pallets" for the three-dimensional cases (3D, all dimensions fixed or infinite height) and "bins", "plates", or "(stock) sheets" (all dimensions fixed) or "strips" (only width fixed, infinite height) in 2D.

Objectives are, for instance, to minimize the number of containers or to maximize the material used (hence to minimize the "trim loss", i.e. the wasted area). A huge number of practical or industrial applications are concerned, such as truck loading, cardboard packing, facilities, fashion, plant, machine, newspaper, or web page layout design, VLSI macro-cell placement, glass, cloth, metal, paper, or wood industries, dynamic memory allocation, meta-computing, multi-processor or publicity scheduling for instance. This may explain why (commercial) software packages exist, sometimes for a long time. See (Dowsland \& Dowsland, 1992; Lodi et al., 2002; Wäscher et al., 2007) just to mention a few surveys.

\footnotetext{
* Corresponding author.

1 Expressions like "(pallet) loading”, “containment", “marker making”, "nesting”, “(layout) design", "placement", "(resource) allocation”, or "(stock) cutting” e.g. are also sometimes employed to refer to this type of problems.
} 
These problems are usually generalizations or restrictions of the well-known NP-hard (or NPcomplete for decision variants) quadratic assignment, bin packing, knapsack, or quadratic set covering problems. Packing problems are thus optimization or satisfaction problems (sometimes with multiple objectives) that are NP-hard or NP-complete in the general case (Fowler et al., 1981; Garey \& Johnson, 1979).

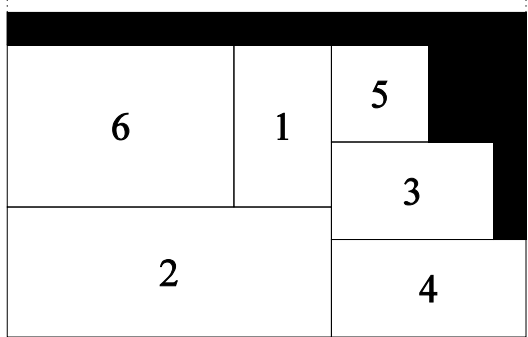

Feasible

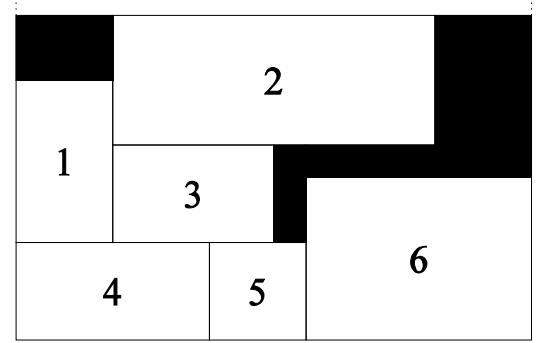

Not feasible

Figure 1. The guillotine constraint imposes a pattern where the items can be extracted by a sequence of "edgeto-edge" cuts, i.e. the cutting tool cannot change of direction within the same cutting step (dark zones map wasted areas).

This paper is dedicated to the NP-hard 2D Strip Packing Problem (2D-SPP) which can be informally stated as follows: Given a finite set of objects, pack all of them without overlap in one strip of an infinite height and fixed width (also called "basis") while minimizing the height of the resulting packing. The guillotine constraint is not considered here. Furthermore, all objects are regular (rectangular to be more precise) and cannot be rotated, i.e. they have a fixed orientation.

In this paper, we introduce CTS (for "Consistent Tabu Search"), a reinforced tabu search algorithm dedicated to the 2D-SPP. Compared with previous algorithms for the 2D-SPP, our CTS has several notable features. First, it handles a consistent neighborhood. Second, CTS evaluates packings, possibly partial, using problem knowledge. Finally, our algorithm includes a diversification mechanism relying on a set of historically "frozen" rectangles. Computational results suggest that CTS may be of great interest to solve the 2D-SPP.

In the two next sections, the 2D-SPP is formally stated and a brief description of various existing methods is given. Section 4 is devoted to the detailed presentation of our dedicated tabu search algorithm for the 2D-SPP. Experimental results are finally shown in Sect. 5 on a set of well-known benchmarks and compared with previous attempts including best performing state-of-the-art algorithms.

\section{PROBLEM FORMULATION}

A "strip" is a 2D vertical space with fixed width $W$ and infinite height, see Figure 2. The bottom-left (BL) corner of the strip stands for the $(0,0)$ point of an xy-plane where the $\mathrm{x}$-axis (respectively $\mathrm{y}$-axis) is the direction of the width (resp. height).

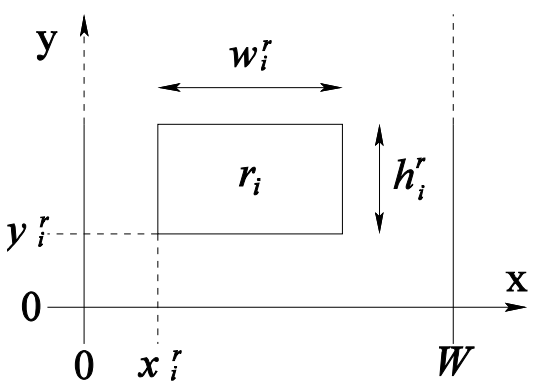

Figure 2. A strip with basic notations. 
The set of $n \geq 2$ Rectangles to be positioned in the strip is $R=\left\{r_{1}, \ldots, r_{n}\right\}$ where the weight (resp. height) of each object $r_{1 \leq i \leq n}$ is $0<w_{i}^{r} \leq W$ (resp. $h_{i}^{r}>0$ ).

According to these notations, the 2D-SPP is then to determine the $\left(x_{i}^{r}, y_{i}^{r}\right)$ coordinates of the BL corner of all rectangles (i.e. the location of each $r_{i} \in R$ in the strip) so as to minimize the height of the resulting packing. This can be formally stated as follows:

$$
\begin{array}{cl}
\text { Minimize } f: & \max _{1 \leq i \leq n}\left\{y_{i}^{r}+h_{i}^{r}\right\} \\
\text { Subject to: } & 0 \leq x_{i}^{r} \leq W-w_{i}^{r} \wedge y_{i}^{r} \geq 0 \\
& \wedge\left(x_{i}^{r} \geq x_{j}^{r}+w_{j}^{r} \vee x_{i}^{r}+w_{i}^{r} \leq x_{j}^{r}\right. \\
& \left.\vee y_{i}^{r} \geq y_{j}^{r}+h_{j}^{r} \vee y_{i}^{r}+h_{i}^{r} \leq y_{j}^{r}\right) .
\end{array}
$$

where (2) forces each rectangle $r_{i}$ to be inside the strip and (3-4) specify that any two $r_{i}$ and $r_{j \neq i}$ objects must not overlap horizontally or vertically.

\section{LITERATURE REVIEW}

Only a few exact methods are available for 2D-SPP or closely related problems (see Sect. 3.1), while a wide range of approximate heuristics has been reported (Sect. 3.2). Among these strategies, the greedy randomized adaptive search procedure from Alvarez-Valdes et al. (2008) and the hybrid hyperheuristic + intensification / diversification walk strategy from Neveu et al. (2008) are probably the best performing ones for 2D-SPP (these two effective methods are briefly summarized in Sect. 3.2).

\subsection{Exact methods}

These approaches are often based on implicit enumeration of the search space. They are thus usually limited to small instances. However, given sufficient time, they can in theory either find a solution (optimal for optimization problems) or prove that none exists (for satisfaction problems).

The branch-and-bound algorithm presented by Martello et al. (2003) relies on a 2D-SPP relaxation (basically, cutting each object $r_{i} \in R$ into items of height lower than $h_{i}^{r}$ ) that can be solved as a particular NP-hard one-dimensional bin-packing problem (with "side" constraints). This leads to a lower bound on $f_{O P T}$, the OPTimum value of (1), better than those previously proposed.

Recently, two branch-and-bound approaches faster than those from Martello et al. (2003) were designed by Kenmochi et al. (2009). Including many components, dynamic programming cuts for instance, the 2D-SPP optimization problem (as formally defined in Sect. 2) is reduced here to the "perfect packing problem" which is a satisfaction problem (determine if a packing without wasted space exists), sometimes by adding new objects.

Other (recent) exact methods related to 2D-SPP, or bounds, can be found e.g. in (Belov et al., 2009; Clautiaux et al., 2008; Soh et al., 2008).

\subsection{Approximate heuristics}

These approaches include e.g. "greedy" constructive strategies or "(meta) heuristics". While they loss the completeness of exact methods, they can handle large instances and usually obtain good quality 
solutions in reasonable time. In this section, we review two best-performing algorithms and discuss about other representative heuristics related to 2D-SPP.

Alvarez-Valdes et al. (2008) proposed a Greedy Randomized Adaptive Search Procedure (GRASP) for 2D-SPP. It is a multi-start scheme that iteratively builds a feasible solution in a greedy way following various dynamic pseudo-random selection rules. This solution is then modified to try to correct previous wrong random choices, for use in the next greedy step, by different "simple local search" algorithms.

Neveu et al. (2008) developed an hybrid approach combining a hyperheuristic $(\mathrm{HH})$ with the intensification / diversification walk strategy (IDW). It starts (HH phase) with a greedy packing, where the selection rule possibly alternates between different criteria, e.g. in a round robin manner. This solution is then perturbed (IDW phase) by iteratively moving an object at the top of the strip below its current location. If such a perturbation generates overlaps, they are repaired using a greedy heuristic (possibly randomly chosen).

Other representative approaches for 2D-SPP (or closely related variants) include, for instance:

- Simulated annealing (Burke et al., 2009; Hopper \& Turton, 2001; Soke \& Bingul, 2006).

- Tabu search (Alvarez-Valdes et al., 2007; Błażewicz et al., 2004; Hamiez et al., 2009; Iori et al., 2003).

- Iterated local search (Imahori et al., 2005, 2003).

- Genetic and evolutionary algorithms (Beasley, 2004; Bortfeldt, 2006; Gómez-Villouta et al., 2008; Gonçalves, 2007; Hopper \& Turton, 2001; Iori et al., 2003; Soke \& Bingul, 2006).

- Hybrid (meta)heuristics (Beltrán Cano et al., 2004; Ibaraki et al., 2008; Iori et al., 2003; Mir \& Imam, 2001; Neveu et al., 2008; Yeung \& Tang, 2004).

- Hyperheuristics (Araya et al., 2008; Garrido \& Riff, 2007; Terashima-Marín et al., 2005).

Note that polynomial-time approximation schemes with (asymptotic or absolute) performance guarantee are also available for these problems (Harren \& van Stee, 2009; Jansen \& van Stee, 2005).

\section{CTS: A CONSISTENT TABU SEARCH FOR 2D-SPP}

We first recall here the fundamentals of tabu search (Sect. 4.1) and how the problem is addressed (Sect. 4.2). Next sections (4.3-4.8) describe then the problem-specific components of our CTS, where all $p$ variables (with subscripts) are parameters whose values will be given in the experimentation part (Sect. 5.1). The general CTS procedure is finally summarized in Sect. 0.

\subsection{A brief review of tabu search}

Tabu search is an advanced metaheuristic designed for tackling hard combinatorial optimization or satisfaction problems (Glover \& Laguna, 1997). It relies on a neighborhood relation as well as some forms of memory and learning strategies to explore effectively a search space. Let $(S, f)$ be our search problem where $S$ and $f$ are respectively the search space and the optimization objective.

A "neighborhood" $N$ over $S$ is any function that associates to each individual $s \in S$ some solutions $N(s) \subset S$. Any solution $s^{\prime} \in N(s)$ is called a neighboring solution or simply a neighbor of $s$. For a given neighborhood $N$, a solution $s$ is a "local optimum" with respect to $N$ if $\mathrm{s}$ is the best among the solutions in $N(s)$. The notion of neighborhood can be explained in terms of the "move" operator. Typically applying a move $\mu$ to a solution $s$ changes slightly $s$ and leads to a neighboring solution $s$ '. 
This transition from a solution to a neighbor is denoted by $s^{\prime}=s \oplus \mu$. Let $\Gamma(s)$ be the set of all possible moves which can be applied to solution $s$, then the neighborhood $N(s)$ of $s$ can be defined by: $N(s)=\{s \oplus \mu: \mu \in \Gamma(s)\}$.

A typical tabu search algorithm begins with an initial configuration in $S$ and proceeds iteratively to visit a series of locally best configurations following the neighborhood. At each iteration, a best neighbor $s^{\prime} \in N(s)$ is sought to replace the current configuration $s$ even if $s^{\prime}$ does not improve $s$ in terms of the cost function.

To avoid the problem of possible cycling and to allow the search to go beyond local optima, tabu search introduces the notion of "tabu list", one of the most important components of the method. A tabu list $\tau$ is a special short term memory that maintains a selective history composed of previously encountered solutions or, more generally, pertinent attributes (or moves) of such solutions. A simple strategy based on this short term memory consists in preventing previously visited solutions from being reconsidered for the next $p_{\tau}$ iterations ( $p_{\tau}$, called "tabu tenure", is problem dependent). Now, at each iteration, tabu search searches for a best neighbor from this dynamically modified neighborhood.

\subsection{Solving scheme}

Let $2 \mathrm{D}-\mathrm{SPP}_{k>0}$ be the following satisfaction problem: Is there a solution $s$ to 2D-SPP such that $f(s) \leq k$ ? Obviously, 2D-SPP is equivalent to find the lowest $k$ such that $2 \mathrm{D}-\mathrm{SPP}_{k}$ holds.

CTS treats the 2D-SPP optimization problem (minimizing the height $f$ ) as successive 2D-SPP ${ }_{k}$. Starting from a complete packing $s_{0}$ of height $f\left(s_{0}\right)$, e.g. obtained with a greedy method (see Sect. 4.4), CTS tackles 2D-SPP $k$ with decreasing values of $f\left(s_{0}\right)$ for $k$. To be more precise, if CTS finds a solution $s$ to $2 \mathrm{D}-\mathrm{SPP}_{k}$, it then tries to solve $2 \mathrm{D}-\mathrm{SPP}_{f(s)-p_{f}}\left(p_{f}>0\right.$, for decrement of the height).

\subsection{Search space: A direct representation}

Some approaches for 2D-SPP, or closely related variants, consider a (quite natural or, at least, intuitive) search space $S$ composed of the set of (all) permutations of the objects, see (Gómez-Villouta et al., 2008; Iori et al., 2003; Soke \& Bingul, 2006; Yeung \& Tang, 2004) for instance.

More precisely, for a given $n$-set $R$ of objects to be packed, a permutation $s \in S$ of $[1, \ldots, n]$ is built (statically or dynamically) using a selection heuristic $\sigma^{2}$ which is followed by a given placement heuristic $\phi$ (or "decoder"). In other words, given a selection operator $\sigma$ and a $\phi$ decoder, one can locate all the objects using $\phi$ and according to the order imposed by $\sigma$, see Algorithm 1 where $s_{\rho}$ is the element at rank $\rho$ in permutation $s$. The problem is then to find a particular permutation $s * \in S$ (from the $n$ ! available) such that the resulting packing is optimal, i.e. $f(s *)=f_{O P T}$.

Note that many (usually greedy) selection / placement heuristics have been investigated according to various criteria (Alvarez-Valdes et al., 2008, 2007; Aşik \& Özcan, 2009; Burke et al., 2009).

CTS does not code packings with permutations but adopts a direct representation where a 2D$\mathrm{SPP}_{k}$ packing $s \in S$ (optimal or not, possibly partial) is a $\{L, E\}$ set $^{3}$ :

\footnotetext{
${ }^{2} \sigma$ introduces then an order for all the objects.

${ }^{3}$ Other approaches that do not use permutations to model the problem, or closely related variants, can also be found e.g. in (Bortfeldt, 2006; Hamiez et al., 2009; Soh et al., 2008).
} 
- $L \subseteq R$ is the set of rectangles properly Located in the strip, i.e. $r_{i}$ verifies (2) with $y_{i}^{r}+h_{i}^{r} \leq k \forall$ $r_{i} \in L$ and $\left(r_{i}, r_{j}\right)$ verifies (3-4) $\forall\left(r_{i}, r_{j \neq i}\right) \in L \times L$. Let the set of "free" objects, i.e. rectangles not (yet) located in the strip, be $\bar{L}=R \backslash L$.

- $E$ is a set of rectangular Empty spaces in the strip. Each empty space $e_{i} \in E$ is characterized by the coordinates $\left(x_{i}^{e}, y_{i}^{e}\right)$ of its BL corner, a width $0<w_{i}^{e} \leq W$, and a height $0<h_{i}^{e} \leq k$, with $0 \leq x_{i}^{e} \leq W-w_{i}^{e}$ and $0 \leq y_{i}^{e} \leq k-h_{i}^{e}$.

Each empty space $e_{i} \in E$ is a maximal rectangle ${ }^{4}$, i.e. $e_{i}$ is not included in another empty space $e_{j}: \forall\left(e_{i}, e_{j \neq i}\right) \in E \times E, x_{i}^{e}<x_{j}^{e} \vee x_{i}^{e}+w_{i}^{e}>x_{j}^{e}+w_{j}^{e} \vee y_{i}^{e}<y_{j}^{e} \vee y_{i}^{e}+h_{i}^{e}>y_{j}^{e}+h_{j}^{e}$.

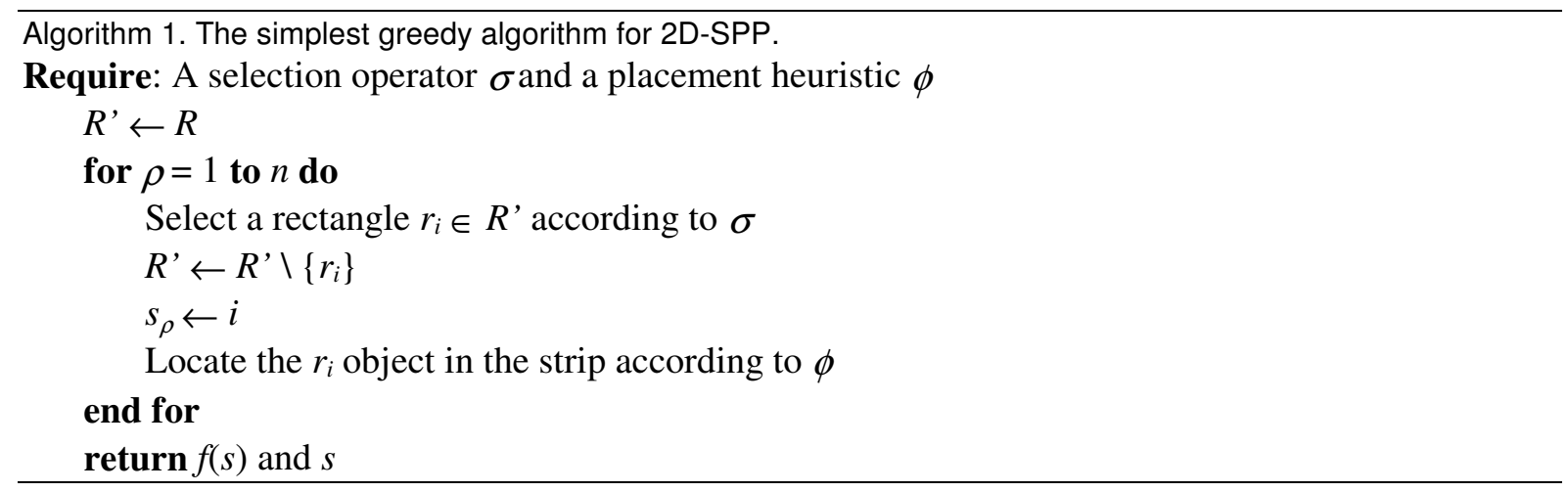

\subsection{Initial configuration}

Tabu search needs an initial configuration $s_{0}$ that specifies where the search begins in the search space $S$. CTS uses Algorithm 1 to construct $s_{0}$, where the $\phi$ placement heuristic is the "Bottom Left Fill" procedure (BLF) from Baker et al. (1980) and the $\sigma$ selection operator orders all rectangles $r_{i} \in R$ first by decreasing width, secondly by decreasing height (when two objects $r_{i}$ and $r_{j \neq i}$ have the same width), randomly last if necessary ( $r_{i}$ and $r_{j \neq i}$ have the same width and height).

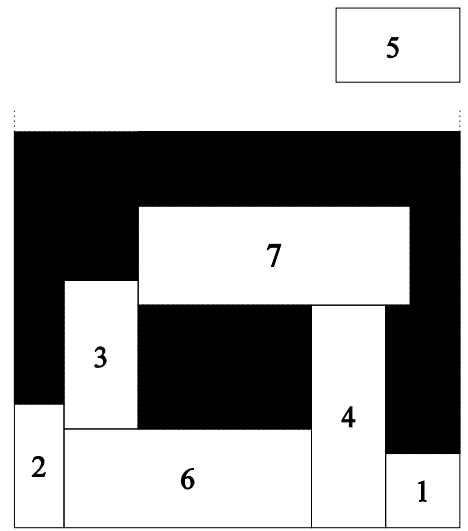

Before BLF

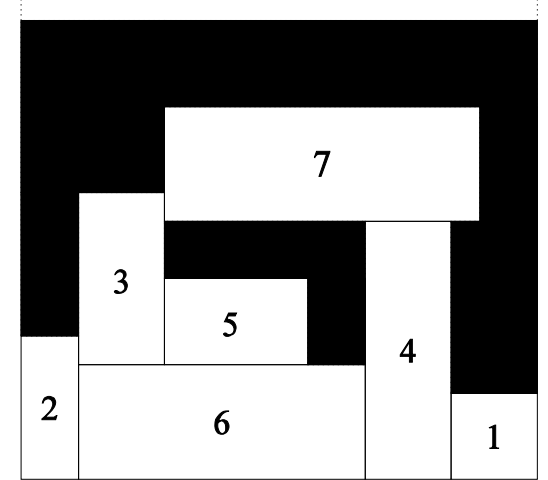

After BLF

Figure 3. Basically, BLF places each object at the left-most and lowest possible free area.

BLF is capable of filling enclosed wasted areas, see Figure 3 where rectangle $r_{5}$ has to be packed. Notice that, according to the way BLF is implemented, its worst time complexity goes from $\mathrm{O}\left(n^{3}\right)$

\footnotetext{
${ }^{4}$ The notion of "maximal rectangular empty space" seems to have been independently introduced in (El Hayek et al., 2008) (where it is called "maximal area") and (Neveu et al., 2008) ("maximal hole"). $|E|$ is at most (i.e. in the worst case) in $\mathrm{O}\left(n^{2}\right)$ according to El Hayek et al. (2008).
} 
(Hopper \& Turton, 2001) to $\mathrm{O}\left(n^{2}\right)$ (Chazelle, 1983) for a permutation of $n$ objects. We employed this decoder / order since some previous experiments (Hopper \& Turton, 2001; Imahori et al., 2007) suggested that the BLF placement algorithm usually outperforms other decoders .

Note that the initial packing $s_{0}$ is a solution to $2 \mathrm{D}-\mathrm{SPP}_{k} \forall k \geq f\left(s_{0}\right)$. So, $s_{0}$ provides a trivial upper bound for 2D-SPP: $f_{O P T} \leq f\left(s_{0}\right)$.

\subsection{Cost function}

This measure, also called "evaluation" or "fitness" function, is a key component of tabu search because it guides the choices of the algorithm at each iteration.

Let the Maximum width and height of free rectangles be respectively $M_{w}=\max _{r_{i} \in \bar{L}}\left\{w_{i}^{r}\right\}$ and $M_{h}=\max _{r_{i} \in \bar{L}}\left\{h_{i}^{r}\right\}$, the number of free objects with width $M_{w}$ be $\alpha=\mid\left\{r_{i} \in \bar{L}: w_{i}^{r}=M_{w}\right\}$, the "density" of a $2{\mathrm{D}-\mathrm{SPP}_{k}}_{k}$ packing be $\delta=\sum_{e_{j} \in E}\left(W-x_{j}^{e}\right)\left(k-y_{j}^{e}\right)^{6}$, and the Maximum area of empty spaces be $M_{a}=\max _{e_{j} \in E}\left\{w_{j}^{e} \cdot h_{j}^{e}\right\}$. CTS uses the $c$ function (for "cost", to be minimized) formally defined by (5) to evaluate a (possibly partial) 2D-SPP ${ }_{k}$ packing $s \in S$, see also Figure 4 for a numerical example.

$$
c(s)= \begin{cases}M_{w} M_{h} \alpha & \text { if } E=\emptyset \\ \frac{M_{w} M_{h} \alpha \delta}{M_{a}} & \text { otherwise } .\end{cases}
$$

Roughly speaking, the value $c(s)$ measures the quality of solution $s$ with respect to 2D-SPP $k$, the current satisfaction problem considered:

- $c(s)=0$ signifies that $s$ is a solution to $2 \mathrm{D}-\mathrm{SPP}_{k}$. Furthermore, $s$ is usually called a "perfect" packing if it does not include an empty space $(E=\varnothing)$. In this case, $s$ is an optimal solution to 2D-SPP (i.e. $2 \mathrm{D}-\mathrm{SPP}_{k^{\prime}}$ admits no solution $\forall k^{\prime}<k$ ): $f_{O P T}=k$.

- $c(s)>0$ indicates a partial packing. Here, $E=\varnothing$ (no empty space) means that $2 \mathrm{D}^{-\mathrm{SPP}_{k^{\prime}} \text { has no }}$ solution $\forall k^{\prime} \leq k$ and that a trivial lower bound has been found for 2D-SPP: $f_{O P T}>k$.

3

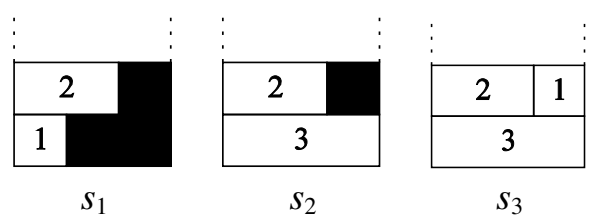

Figure 4. Let $r_{1}$ be the unit square (i.e. $w_{1}^{r}=h_{1}^{r}=1$ ) and $k=2$. $c\left(s_{1}\right)=9$ since $E \neq \varnothing, M_{w}=w_{3}^{r}=3$, $M_{h}=h_{3}^{r}=1, \alpha=1, \delta=(3-1)(2-0)+(3-2)(2-0)=6$, and $M_{a}=2$. Similarly, $c\left(s_{2}\right)=1$ since $E \neq \varnothing, M_{w}=1$, $M_{h}=1, \alpha=1, \delta=1$, and $M_{a}=1 . c\left(s_{3}\right)=0$ since $E=\varnothing$ and $\alpha=0$.

\footnotetext{
${ }^{5}$ However, note that while BLF has an asymptotic performance of $3 f_{O P T}$, other heuristics achieved better (asymptotic or absolute) performance ratio: 2.7 (Coffman Jr. et al., 1980), 2 (Schiermeyer, 1994), $1+\varepsilon$ (Baker et al., 1981).

${ }^{6} \mathrm{~A}$ "small" $\delta$ value indicates that (almost) all empty spaces are concentrated close to the top-right corner of the strip.
} 
The cost and objective functions, $c$ (5) and $f(1)$ respectively, are used to compare any two packings $s_{1}$ and $s_{2}$ (possibly partial): With respect to $2 \mathrm{D}-\mathrm{SPP}_{k}, s_{1}$ is said to be "better" than $s_{2}$ if the evaluation of $s_{1}$ is lower than that of $s_{2}$, formally $c\left(s_{1}\right)<c\left(s_{2}\right)$. However, note that the $c$ fitness function is inadequate when $s_{1}$ and $s_{2}$ are both solutions to $2 \mathrm{D}-\mathrm{SPP}_{k}$, i.e. when $c\left(s_{1}\right)=c\left(s_{2}\right)=0$. In this case, $s_{1}$ is better than $s_{2}$ if $f\left(s_{1}\right)<f\left(s_{2}\right)$.

Other evaluation functions have been proposed for 2D-SPP. Neveu et al. (2007) consider the number $u$ of " $u$ nits filled by rectangles on the highest line of the strip" to define $c_{N}(s)=(f(s)-1) W+$ $u$. Hamiez et al. (2009) proposed a similar fitness function, relying on the set $\lceil R\rceil \subseteq R$ of rectangles at the top of the strip, formally defined by (6) where $\lceil R\rceil=\left\{r_{i} \in R: y_{i}^{r}+h_{i}^{r}>H_{*}-p_{H}\right\}, p_{H}$ is an integer parameter, and $H_{*}$ is the best height found, initially the height $f\left(s_{0}\right)$ of the starting solution $s_{0}$. One can observe that $c_{N}$ and $c_{H}$ compute a measure solely based on rectangles at the top of the strip and do not consider what happens below these rectangles. Our $c$ cost function seems thus more relevant since it precisely includes a measure (the $\delta$ component) related to this useful information.

$$
c_{H}(s)=\left\{\begin{array}{l}
0 \text { if }\lceil R\rceil=\emptyset \\
\sum_{r_{i} \in\lceil R\rceil} w_{i}^{r} *\left(y_{i}^{r}+h_{i}^{r}-H_{*}+p_{H}\right) \text { otherwise } .
\end{array}\right.
$$

\subsection{Neighborhood}

The neighborhood $N$ is another key element of tabu search. It defines a structure of the search space $S$ and determines the paths the algorithm will follow to explore $S$.

The main goal of the CTS neighborhood is to empty the set of free objects, i.e. the set $\bar{L}$ of rectangles not yet placed in the strip. Basically, it tries to locate a free rectangle $r_{i}$ in the strip $\left(r_{i}\right.$ moves then from $\bar{L}$ to $L$ ), at the BL corner either of an empty space (defining a sub-neighborhood $N_{E}$, described in Sect. 4.6.1) or of another placed object (defining $N_{L}$, Sect. 4.6.2).

This location for object $r_{i}$ may generate overlaps with a set $L_{i} \subseteq L$ of other rectangles already in the strip: $\quad L_{i}=\left\{r_{j \neq i} \in L: x_{i}^{r}<x_{j}^{r}+w_{j}^{r} \wedge x_{i}^{r}+w_{i}^{r}>x_{j}^{r} \wedge y_{i}^{r}<y_{j}^{r}+h_{j}^{r} \wedge y_{i}^{r}+h_{i}^{r}>y_{j}^{r}\right\}$. All objects overlapping with the $r_{i}$ rectangle are thus removed from the strip to repair these overlaps $\left(\forall r_{j} \in L_{i}, r_{j}\right.$ moves from $L$ to $\bar{L}$ ). This principle, known as "ejection chains", is used to make the neighboring configuration consistent with (3-4).

Finally, notice that locating a rectangle in the strip and the possible ejection of all overlapping objects imply updates of the set of empty spaces $(E)$. This is done using the efficient "incremental" procedures detailed in (Neveu et al., 2008).

\subsubsection{Neighborhood $N_{E}$ : Consider the empty spaces}

CTS examines two cases here, with two different objectives: To reduce the number of free rectangles, i.e. to minimize $|\bar{L}|$ (defining $N_{E}^{|\bar{L}|}$ ), or to make the packing $\operatorname{Denser}\left(N_{E}^{D}\right)$.

The case of $N_{E}^{|\bar{L}|}$. E . All free objects $r_{i} \in \bar{L}$ are tried to be located in the strip to the BL corner of all empty spaces $e_{j} \in E$ such that $r_{i}$ fits entirely in $e_{j}$. More formally, $r_{i}$ and $e_{j}$ must verify $x_{j}^{e}+w_{i}^{r} \leq W \wedge y_{j}^{e}+h_{i}^{r} \leq k \wedge w_{j}^{e} \geq w_{i}^{r} \wedge h_{j}^{r} \geq h_{i}^{r}$. This generates $|\bar{L}|$ sets $N_{E}^{|\bar{L}|}(s, i)$ of 
neighbors for configuration $s$ (some possibly empty), $N_{E}^{|\bar{L}|}(s)$ being the union of these sets: $N_{E}^{|\bar{L}|}(s)=\bigcup_{r_{i} \in \bar{L}} N_{E}^{|\bar{L}|}(s, i)$.

Note that $N_{E}^{|\bar{L}|}(s) \neq \varnothing$ means that there is at least one free rectangle $r_{i} \in \bar{L}$ and one empty space $e_{j} \in E$ such that $r_{i}$ fits in $e_{j}$, i.e. that the number of free objects can be reduced. Furthermore, in this case, locating $r_{i}$ will generate no overlap $\left(L_{i}=\varnothing\right)$, hence no repairing is needed.

The case of $N_{E}^{D}$. Here again, all free rectangles $r_{i} \in \bar{L}$ are tried to be located to the BL corner of all empty spaces $e_{j} \in E$ but the previous condition on $e_{j}$ and $r_{i}$ is relaxed to allow overlaps. More formally, $r_{i}$ and $e_{j}$ must only verify $x_{j}^{e}+w_{i}^{r} \leq W \wedge y_{j}^{e}+h_{i}^{r} \leq k$.

Note that $N_{E}^{D}(s)=\varnothing$ means either, $\forall\left(r_{i}, e_{j}\right) \in \bar{L} \times E$, that the empty space $e_{j}$ is located rather to the right (top, respectively) of the strip and placing the $r_{i}$ rectangle in $e_{j}$ will violate (2) since $x_{j}^{e}+w_{i}^{r}>W$ (resp. will not follow the definition of $2 \mathrm{D}-\mathrm{SPP}_{k}$ since $y_{j}^{e}+h_{i}^{r}>k$ ) or that it is forbidden to remove at least one rectangle overlapping with the $r_{i}$ object (this is due to some restrictions described in Sect. 0).

\subsubsection{Neighborhood $N_{L}$ : Consider all rectangles already packed}

From the current configuration $s$, all free objects $r_{i} \in \bar{L}$ are tried to be located to the BL corner of all already packed rectangles $r_{j} \in L$ such that $r_{i}$ and $r_{j}$ have different sizes, locating $r_{i}$ will respect (2), and the resulting packings will follow the definition of 2D-SPP ${ }_{k}$. More formally, $r_{i}$ and $r_{j}$ must verify $\left(w_{i}^{r} \neq w_{j}^{r} \vee h_{i}^{r} \neq h_{j}^{r}\right) \wedge x_{j}^{r}+w_{i}^{r} \leq W \wedge y_{j}^{r}+h_{i}^{r} \leq k$.

This generates $|\bar{L}|$ sets $N_{L}(s, i)$ of neighbors for the configuration $s$ with $0 \leq\left|N_{L}(s, i)\right| \leq|L|: N_{L}(s)$ is the union of these sets. Similarly to $N_{E}^{D}$, note that $N_{L}(s)=\varnothing$ means that all overlapping objects cannot be removed from the strip (see Sect. 0).

\subsubsection{Search strategy}

Our neighborhood $N$ is composed of the three sub-neighborhoods described above: $N_{E}^{|\bar{L}|}$ and $N_{E}^{D}$ (Sect. 4.6.1), and $N_{L}$ (Sect. 4.6.2). They are explored by CTS in a hierarchical way: $N_{E}^{|\bar{L}|}$ is always tried the first, then $N_{E}^{D}$ is used only if $N_{E}^{|\bar{L}|}$ is not applicable (i.e. empty), $N_{L}$ being (possibly) considered the last. This scheme is more formally detailed in Algorithm 2.

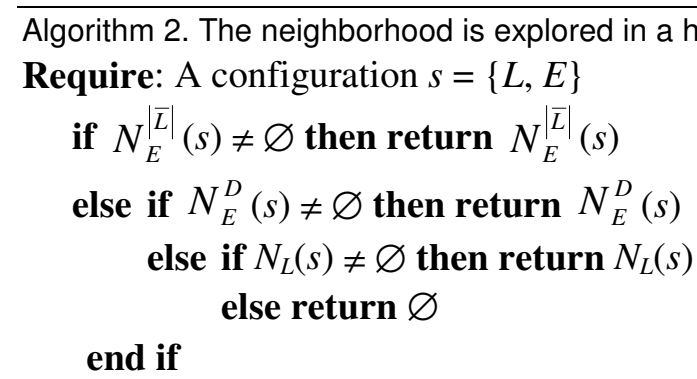


Note that if configuration $s$ has no neighbor, i.e. $N(s)=N_{E}^{|\bar{L}|}(\mathrm{s})=N_{E}^{D}(\mathrm{~s})=N_{L}(\mathrm{~s})=\varnothing$, no move is performed and diversification is invoked (see Sect. 4.8).

\subsection{Tabu list}

At current iteration $m$, since a CTS move from solution $s$ to a neighbor $s^{\prime} \in N(s)$ consists in locating one free rectangle $r_{i} \in \bar{L}$ in the strip, it seems quite natural to forbid object $r_{i}$ leaving the strip from configuration $s$ '. This "reverse" move will then be stored in the tabu list $\tau$ for a duration $0<p_{\tau} \leq n$ (integer) to indicate that $r_{i}$ cannot be removed from the strip at least up to iteration $m+p_{\tau}$.

Note that the tabu list $\tau$ is made empty at the beginning of the search or when CTS finds a solution to the current satisfaction problem considered $\left(2 \mathrm{D}-\mathrm{SPP}_{k}\right)$, i.e. if there is no free rectangle at all $(\bar{L}=\varnothing)$.

\subsection{Diversification}

Let $s *$ be the overall best complete packing, according to (1), found by CTS at iteration $m_{*}$ (initially $s *$ is the initial configuration $s_{0}$ introduced in Sect. 4.4 with $m_{*}=0$ ).

When a solution $s$ has no neighbor (i.e. $N(s)=\varnothing$ ) or $s *$ keeps unchanged for a number $p^{*}>0$ of iterations, CTS first resets the tabu list $\tau$ and reloads $s *$ in $s(s \leftarrow s * \tau \leftarrow \varnothing)$. This new current complete packing $s$ is then perturbed according to two different $D$ iversification schemes called $D_{I}$ (for "Interchange", performed with probability $p_{D}$ ) and $D_{T}$ (for "Tetris-like", probability $1-p_{D}$ ). After perturbation, $p_{*}$ supplementary moves are given to CTS to update the overall best complete packing $s *$

\subsection{1 $D_{l}$ : A basic perturbation}

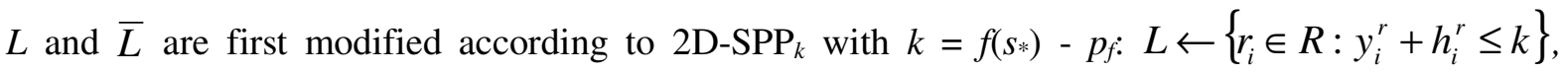
$\bar{L} \leftarrow R \backslash L$. Diversification $D_{I}$ considers then all $\left(r_{i}, r_{j}\right) \in L \times \bar{L}$ such that the $r_{i}$ packed rectangle and the $r_{j}$ free object have different sizes, more formally $r_{i}$ and $r_{j}$ verify $\left(w_{i}^{r} \neq w_{j}^{r} \vee h_{i}^{r} \neq h_{j}^{r}\right) \wedge$ $x_{i}^{r}+w_{j}^{r} \leq W \wedge y_{i}^{r}+h_{j}^{r} \leq k$. It simply interchanges two such elements (randomly selected) and makes $r_{j}$ tabu.

Note that locating the free object $r_{j}$ at the place of $r_{i}$ (i.e. swapping $r_{j}$ from $\bar{L}$ to $L$ and $r_{i}$ from $L$ to $\bar{L}$ ) may cause overlaps. In this case, repairing is done like in Sect. 4.6.

\subsection{2 $D_{T}$ : A perturbation based on the history}

During the overall search process, CTS keeps for each rectangle $r_{i} \in R$ the number $F_{i}$ (for "Frequency") of times $r_{i}$ leaved the strip, i.e. the number of times $r_{i}$ swaps from $L$ to $\bar{L}^{7}$.

The $D_{T}$ diversification scheme considers a $\pi_{F}$ permutation that orders all objects $r_{i} \in R$ first by increasing frequencies, secondly by decreasing widths (when $F_{i}=F_{j \neq i}$ ), then by decreasing heights $\left(w_{i}^{r}=w_{j \neq i}^{r}\right)$, randomly last if necessary $\left(h_{i}^{r}=h_{j \neq i}^{r}\right)$. Let the set $\lfloor F\rfloor$ be composed of the first $p_{\tau}$ elements of $\pi_{F}$.

\footnotetext{
${ }^{7} F_{1 \leq i \leq n}=0$ at the beginning of the search.
} 
All rectangles $r_{i} \in\lfloor F\rfloor$ are first temporarily removed from the strip and their frequencies are updated $^{8}$. Then, the partial packing is pushed down to the basis of the strip, like in the famous Tetris game. Finally, all objects $r_{i} \in\lfloor F\rfloor$ are sorted like in Sect. 4.4 and relocated in the strip with BLF, see Figure 5.

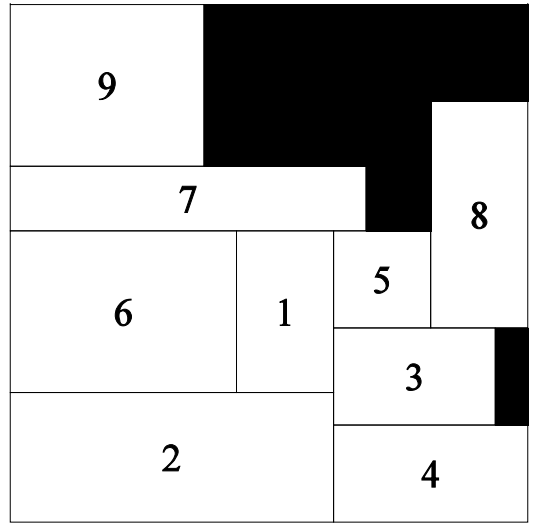

Let $\lfloor F\rfloor=\left\{r_{1}, r_{2}, r_{3}, r_{5}\right\}$

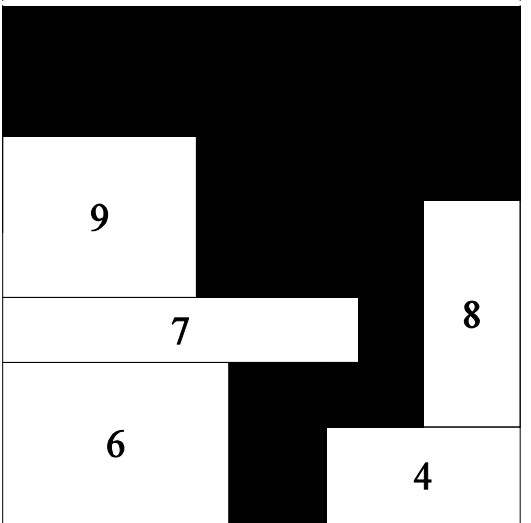

Unpack $\lfloor F\rfloor$ and push down

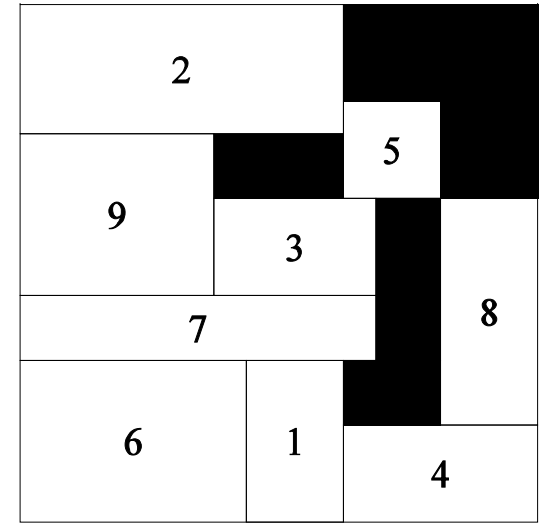

Repack $\lfloor F\rfloor$

Figure 5. The $D_{T}$ diversification. Surprisingly, experiments showed that almost all rectangles with lowest frequency $\left(F_{i}\right)$ were located close to the bottom of the strip.

CTS deals now with 2D-SPP ${ }_{k}$ with $k=f(s)-p_{f}: L \leftarrow\left\{r_{i} \in R: y_{i}^{r}+h_{i}^{r} \leq k\right\}, \bar{L} \leftarrow R \backslash L$. This means that CTS possibly considers $2 \mathrm{D}-\mathrm{SPP}_{k}$ problems with $k \geq f\left(s_{0}\right) \geq f\left(s_{*}\right)$.

\subsection{CTS: The general procedure}

Algorithm 3. An overview of CTS.

Require: A starting configuration $s=\{L, E\}$ such that $L=R / /$ Hence $\bar{L}=\varnothing$

1. $m \leftarrow 0, s * \leftarrow s, m_{*} \leftarrow 0 / /$ Initialization

2. while $m \leq p_{M}$ do

3. if $\bar{L}=\varnothing$ then

4. $\quad$ if $f(s)<f(s *)$ or $\left(f(s)=f(s *)\right.$ and some probability $p_{\approx}$ is verified) then $s * \leftarrow s, m_{*} \leftarrow m$

5. $L \leftarrow\left\{r_{i} \in R: y_{i}^{r}+h_{i}^{r} \leq f(s)-p_{f}\right\}, \bar{L} \leftarrow R \backslash L$

6. $\quad \tau \leftarrow \varnothing / /$ Tabu list

7. $\quad$ if $N(s)=\varnothing$ then $D i v \leftarrow$ true // To Diversify

8. $\quad$ else

9. $\quad m \leftarrow m+1$

10. Let $\lfloor N(s)\rfloor$ be the set of the best evaluated neighbors $s$ ' of $s$ according to (5): $\lfloor N(s)\rfloor=\left\{s_{1}^{\prime} \in N(s): \forall s_{2}^{\prime} \in N(s), c\left(s_{1}^{\prime}\right) \leq c\left(s_{2}^{\prime}\right)\right\}$

11. $\quad$ if $c\left(s^{\prime}\right)>0 \forall s^{\prime} \in\lfloor N(s)\rfloor$ then

12. $\quad$ if $\left(m-m_{*}\right) \bmod p_{*}=0$ then $\operatorname{Div} \leftarrow$ true else $D i v \leftarrow$ false

13. Select $s^{\prime} \in\lfloor N(s)\rfloor$ at random

14. $\quad$ else $D i v \leftarrow$ false, select $s^{\prime} \in\lfloor N(s)\rfloor$ minimizing (1) at random

15. $\quad$ if $\operatorname{Div}$ then $s \leftarrow s *, \tau \leftarrow \varnothing$, modify $s$ using $D_{I}$ or $D_{T}$ according to $p_{D} / /$ Diversification

16. $\quad$ else $s \leftarrow s$, update $\tau$

17. return $f(s *)$ and $s *$

\footnotetext{
${ }^{8} F_{i} \leftarrow 2 F_{i}$ is used here to avoid considering (almost) the same $\lfloor F\rfloor$ set in next applications of diversification $D_{T}$ while $F_{i} \leftarrow F_{i}+1$ is applied when performing a move.
} 
The CTS procedure is summarized in Algorithm 3. It requires an initial complete packing (Sect. 4.4). Then CTS proceeds iteratively to solve a series of 2D-SPP ${ }_{k}$ satisfaction problems. If it finds a solution

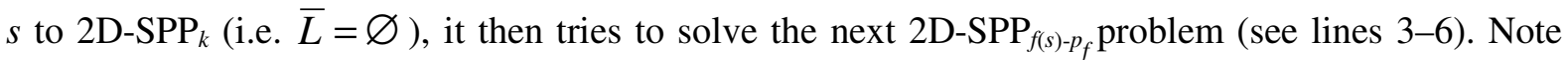
that $f(s)=f(s *)$ (line 4) may occur only after diversification $D_{T}$ (see Sect. 4.8) or at the beginning of the search process.

While it is not mentioned here for simplicity, note that CTS can also end before reaching the $p_{M} \geq 0$ Maximum number of allowed moves (line 2). This may occur each time the overall best complete packing $s *$ is updated (lines 1 and 4 ) whenever the optimum height $f_{O P T}$ (or an upper bound) is known and $f\left(s_{*}\right) \leq f_{O P T}$.

\section{EXPERIMENTATIONS}

We use the complete set of the 21 well-known (perfect) instances defined in (Hopper \& Turton, $2001)^{9}$ to assess the performance of our CTS algorithm. These instances (or a subset of them) are largely studied in the literature. The main characteristics of these instances, including their known optimal height $f_{O P T}$, are given in Table 1 .

Table 1. Main characteristics of the test problems defined in (Hopper \& Turton, 2001). These instances are grouped by "categories" according to the $f_{O P T}$ value.

\begin{tabular}{ccccc}
\hline Category & Instances & $W$ & $n$ & $f_{\text {OPT }}$ \\
\hline C1 & C1P1, C1P2, C1P3 & 20 & $16,17,16$ & 20 \\
C2 & C2P1, C2P2, C2P3 & 40 & 25 & 15 \\
C3 & C3P1, C3P2, C3P3 & 60 & $28,29,28$ & 30 \\
C4 & C4P1, C4P2, C4P3 & 60 & 49 & 60 \\
C5 & C5P1, C5P2, C5P3 & 60 & 73 & 90 \\
C6 & C6P1, C6P2, C6P3 & 80 & 97 & 120 \\
C7 & C7P1, C7P2, C7P3 & 160 & $196,197,196$ & 240 \\
\hline
\end{tabular}

Notice that the instances of categories $\mathrm{C} 4-\mathrm{C} 7$ are very difficult because, to our knowledge, none algorithm is known to be able to optimally solve them. Indeed, some studies don't even report computational results for them, perhaps due to the (very) large size of these instances. Furthermore, the difficulty sometimes also hold for small size instances from categories C1-C3. In particular, various studies report more computational effort for $\mathrm{C} 1 \mathrm{P} 2, \mathrm{C} 2 \mathrm{P} 2$, or $\mathrm{C} 3 \mathrm{P} 2$ or never reach (or are more distant from) an optimal solution for these three specific instances compared to the other similar instances within the same categories.

\subsection{Experimentation conditions}

CTS is coded in the C programming language ("gcc" compiler). All computational results were obtained running CTS on a computer equipped with a $2.83 \mathrm{Ghz}$ quad-core Intel $\circledast$ Xeon $\circledast$ E5440 processor and $8 \mathrm{~Gb} \mathrm{RAM}^{10}$. The values of the CTS parameters are:

- $p_{f}=1$. To build the starting configuration of $2 \mathrm{D}-\mathrm{SPP}_{k}$, the current satisfaction problem considered.

- $p_{\approx} \in[0.4, \ldots, 0.8]$. Probability that a complete packing $s$ replaces the overall best complete packing $s *$, according to (1), whenever $f(s)=f(s *)$.

\footnotetext{
${ }^{9}$ They are available from the "PackLib2" benchmarks library e.g., see http://www.ibr.cs.tu-bs.de/alg/packlib/ $\mathrm{xml} / \mathrm{ht}$-eimhh-01-xml.shtml.

${ }^{10}$ For indicative purpose, the mean running time of CTS ranges from a few seconds (for the smallest instances) to about 33 hours (for the largest instances).
} 
- $p_{\tau} \in[2, \ldots, 6]$. Tabu tenure.

- $p_{*} \in[200, \ldots, 500]$. Maximum number of moves to update $s *$

- $p_{D} \in[0.7, \ldots, 1]$. Probability to apply diversification $D_{I}$.

- $p_{M} \in[1000000, \ldots, 20000$ 000]. Maximum number of allowed moves per run.

The comparison is based on the percentage gap $\gamma$ of a solution $s$ from the optimum or its best bound $\left(f_{O P T}\right): \not(s)=100\left(1-f_{O P T} / f(s)\right)$. The lower is $\chi(s)$, the better is the solution $s$. For CTS, mean gap $\bar{\gamma}$ (resp. best gap $\gamma$ ) is averaged over 5 runs (resp. over best runs only).

\subsection{Computational results}

CTS is compared in Table 2 with five state-of-the-art algorithms that deal with the whole set of instances, or at least categories C1-C6.

We consider two Tabu Search procedures, denoted as TS1 (Iori et al., 2003) ${ }^{11}$ and TS2 (Hamiez et al., 2009), one of the most effective Genetic Algorithm (GA) from Bortfeldt (2006), and two best performing state-of-the-art approaches: The Greedy Randomized Adaptive Search Procedure (GRASP) from Alvarez-Valdes et al. (2008) and the hybrid HyperHeuristic + Intensification / Diversification Walk strategy (HH+IDW) from Neveu et al. (2008).

In Table 2, “-” marks (for HH+IDW, GA, or TS1) mean either that $\bar{\gamma}$ or $\gamma$. cannot be computed or that we did not found the information in (Bortfeldt, 2006; Iori et al., 2003; Neveu et al., 2008). "Mean $\mathrm{C} i$ " are averaged values on category $\mathrm{C} i$. The " $\mathrm{C} 1-\mathrm{C} j$ " aggregated lines, reporting averaged values for all instances in categories $\mathrm{C} 1$ to $\mathrm{C} j$, can be used to identify up to which problem size a particular approach may be effective. The last line shows the number of instances optimally solved.

According to Table 2, TS1 is the worst performing (tabu search) approach for the benchmark tried. Indeed, $\gamma=0$ only for $\mathrm{C} 2 \mathrm{P} 1$ and $\mathrm{C} 2 \mathrm{P} 3$ while the other methods (possibly except GA) always solved at least 8 instances. Almost all other approaches (except TS1 and GA) solved the $\mathrm{C} 1$ and $\mathrm{C} 2$ instances, see line "C1-C2" where $\gamma=0.00$ or $\bar{\gamma}=0.00$.

To our knowledge, the only approaches solving all the 9 instances C1P1-C3P3 are the tabu search from Alvarez-Valdes et al. (2007) and the recent exact procedures described in (Kenmochi et al., 2009; Soh et al., 2008). In Table 2, CTS is the only method reaching the same qualitative results, see line "C1-C3" where $\gamma=0.00$ just for CTS. Furthermore, note that CTS achieves here the lowest $\bar{\gamma}$ value $(0.29<0.36<0.89<1.64<2.69)$.

Aggregated results show that CTS compares also well with the competitors if one considers instances up to C5. Indeed, line "C1-C4" indicates better CTS values for $\gamma *(0.41<0.68 \leq 0.68 \leq$ $0.68<2.38<5.40)$ and $\bar{\gamma}(0.63<0.68<1.08<1.84<2.90)$. The same observation holds in line "C1-C5" only for $\gamma(0.62<0.76 \leq 0.76 \leq 0.76<2.20<5.31)$ but the difference is sharp between the best $\bar{\gamma}(0.76$ for GRASP) and that of CTS (0.85).

CTS obtains worst $\gamma$ or $\bar{\gamma}$ values than those of the two best-known state-of-the-art approaches considered here (GRASP and HH+IDW) only when adding the largest two categories of instances. For $\mathrm{C} 1-\mathrm{C} 6$, note, however, that the difference is still reasonable considering $\gamma *(0.83-0.77=0.06)$.

\footnotetext{
${ }^{11}$ TS1 is perhaps the first tabu search approach for 2D-SPP.
} 
Table 2. Mean and best percentage gaps ( $\bar{\gamma}$ and $\gamma$ resp.) on instances from Hopper \& Turton (2001).

\begin{tabular}{|c|c|c|c|c|c|c|c|c|c|c|c|}
\hline \multirow{2}{*}{ Instances } & \multicolumn{2}{|c|}{ CTS } & \multirow{2}{*}{$\begin{array}{c}\text { TS1 } \\
\gamma \cdot\end{array}$} & \multicolumn{2}{|c|}{ TS2 } & \multicolumn{2}{|c|}{ GA } & \multicolumn{2}{|c|}{ GRASP } & \multicolumn{2}{|c|}{$\mathrm{HH}+\mathrm{IDW}$} \\
\hline & $\bar{\gamma}$ & $\gamma$ & & $\bar{\gamma}$ & $\gamma$ & $\bar{\gamma}$ & $\gamma$. & $\bar{\gamma}$ & $\gamma$ & $\bar{\gamma}$ & $\gamma$ \\
\hline C1P1 & 0.00 & 0.00 & 9.09 & 0.00 & 0.00 & - & - & 0.00 & 0.00 & - & - \\
\hline $\mathrm{C} 1 \mathrm{P} 2$ & 0.00 & 0.00 & 9.09 & 4.76 & 0.00 & - & - & 0.00 & 0.00 & - & - \\
\hline C1P3 & 0.00 & 0.00 & 4.76 & 0.00 & 0.00 & - & - & 0.00 & 0.00 & - & - \\
\hline Mean C1 & 0.00 & 0.00 & 7.65 & 1.59 & 0.00 & 1.59 & 1.59 & 0.00 & 0.00 & 0.48 & 0.00 \\
\hline $\mathrm{C} 2 \mathrm{P} 1$ & 0.00 & 0.00 & 0.00 & 0.00 & 0.00 & - & - & 0.00 & 0.00 & - & - \\
\hline $\mathrm{C} 2 \mathrm{P} 2$ & 0.00 & 0.00 & 6.25 & 0.00 & 0.00 & - & - & 0.00 & 0.00 & - & - \\
\hline $\mathrm{C} 2 \mathrm{P} 3$ & 0.00 & 0.00 & 0.00 & 0.00 & 0.00 & - & - & 0.00 & 0.00 & - & - \\
\hline Mean C2 & 0.00 & 0.00 & 2.08 & 0.00 & 0.00 & 3.33 & 2.08 & 0.00 & 0.00 & 1.87 & 0.00 \\
\hline C3P1 & 0.00 & 0.00 & 3.23 & 0.00 & 0.00 & - & - & 0.00 & 0.00 & - & - \\
\hline $\mathrm{C} 3 \mathrm{P} 2$ & 2.60 & 0.00 & 9.09 & 3.23 & 3.23 & - & - & 3.23 & 3.23 & - & - \\
\hline $\mathrm{C} 3 \mathrm{P} 3$ & 0.00 & 0.00 & 9.09 & 0.00 & 0.00 & - & - & 0.00 & 0.00 & - & - \\
\hline Mean C3 & 0.87 & 0.00 & 7.14 & 1.08 & 1.08 & 3.16 & 3.16 & 1.08 & 1.08 & 2.58 & 1.08 \\
\hline C4P1 & 1.64 & 1.64 & 6.25 & 1.64 & 1.64 & - & - & 1.64 & 1.64 & - & - \\
\hline $\mathrm{C} 4 \mathrm{P} 2$ & 1.64 & 1.64 & 4.76 & 1.64 & 1.64 & - & - & 1.64 & 1.64 & - & - \\
\hline $\mathrm{C} 4 \mathrm{P} 3$ & 1.64 & 1.64 & 3.23 & 1.64 & 1.64 & - & - & 1.64 & 1.64 & - & - \\
\hline Mean C4 & 1.64 & 1.64 & 4.75 & 1.64 & 1.64 & 3.52 & 2.70 & 1.64 & 1.64 & 2.43 & 1.64 \\
\hline C5P1 & 2.17 & 2.17 & 5.26 & 1.10 & 1.10 & - & - & 1.10 & 1.10 & - & - \\
\hline $\mathrm{C} 5 \mathrm{P} 2$ & 1.96 & 1.10 & 3.23 & 1.10 & 1.10 & - & - & 1.10 & 1.10 & - & - \\
\hline C5P3 & 1.10 & 1.10 & 6.25 & 1.10 & 1.10 & - & - & 1.10 & 1.10 & - & - \\
\hline Mean C5 & 1.74 & 1.46 & 4.91 & 1.10 & 1.10 & 2.03 & 1.46 & 1.10 & 1.10 & 1.78 & 1.10 \\
\hline C6P1 & 1.80 & 1.64 & 4.76 & 1.64 & 0.83 & - & - & 1.56 & 0.83 & - & - \\
\hline $\mathrm{C}_{6} \mathrm{P} 2$ & 2.44 & 2.44 & 3.23 & 0.83 & 0.83 & - & - & 1.56 & 0.83 & - & - \\
\hline C6P3 & 2.28 & 1.64 & 3.23 & 1.64 & 0.83 & - & - & 1.56 & 0.83 & - & - \\
\hline Mean C6 & 2.17 & 1.91 & 3.74 & 1.37 & 0.83 & 1.72 & 1.64 & 1.56 & 0.83 & 1.75 & 1.10 \\
\hline C7P1 & 2.20 & 2.04 & - & 1.23 & 1.23 & - & - & 1.64 & 1.64 & - & - \\
\hline $\mathrm{C} 7 \mathrm{P} 2$ & 2.04 & 2.04 & - & 1.23 & 1.23 & - & - & 1.19 & 0.83 & - & - \\
\hline C7P3 & 2.20 & 2.04 & - & 1.23 & 1.23 & - & - & 1.23 & 1.23 & - & - \\
\hline Mean C7 & 2.15 & 2.04 & - & 1.23 & 1.23 & 1.52 & 1.23 & 1.36 & 1.23 & 1.42 & 1.10 \\
\hline $\mathrm{C} 1-\mathrm{C} 2$ & 0.00 & 0.00 & 4.86 & 0.79 & 0.00 & 2.46 & 1.84 & 0.00 & 0.00 & 1.18 & 0.00 \\
\hline C1-C3 & 0.29 & 0.00 & 5.62 & 0.89 & 0.36 & 2.69 & 2.28 & 0.36 & 0.36 & 1.64 & 0.36 \\
\hline $\mathrm{C} 1-\mathrm{C} 4$ & 0.63 & 0.41 & 5.40 & 1.08 & 0.68 & 2.90 & 2.38 & 0.68 & 0.68 & 1.84 & 0.68 \\
\hline C1-C5 & 0.85 & 0.62 & 5.31 & 1.08 & 0.76 & 2.73 & 2.20 & 0.76 & 0.76 & 1.83 & 0.76 \\
\hline C1-C6 & 1.07 & 0.83 & 5.04 & 1.13 & 0.77 & 2.57 & 2.11 & 0.90 & 0.77 & 1.82 & 0.82 \\
\hline $\mathrm{C} 1-\mathrm{C} 7$ & 1.22 & 1.01 & - & 1.14 & 0.84 & 2.41 & 1.98 & 0.96 & 0.84 & 1.76 & 0.86 \\
\hline$\#$ forT $/ 21$ & & & 2 & & & & & & & & \\
\hline
\end{tabular}

\section{CONCLUSIONS}

In this paper, we presented CTS, a Consistent Tabu Search algorithm for the 2D Strip Packing Problem (2D-SPP). CTS includes some components already used by (or, at least, similar to) other approaches, such as the direct representation of the problem or the neighborhood mentioned e.g. in (Hamiez et al., 2009). Apart from these traditional components, our CTS approach was reinforced mainly by introducing two novel elements that, to our knowledge, were never tried for 2D-SPP:

- A fitness function including a measure related to the empty spaces. This was motivated by the fact that most of the previous studies on 2D-SPP usually employ evaluation functions solely based on the rectangles. This is the case, for instance, in (Hamiez et al., 2009; Neveu et al., 2008). Such an additional criterion may be helpful to guide more efficiently the search process.

- A diversification scheme based on a frequency measure $\left(D_{T}\right)$. The motivation behind this component of the generic tabu search strategy, that can help to escape from local optima e.g., originates from running profile observations. Indeed, preliminary tests (without diversification $D_{T}$ ) shown that some rectangles were almost always in the strip and, so, their location changed rarely. $D_{T}$ was thus designed to force these "frozen" objects, that may be considered as problematic (since, perhaps, they are not positioned there in optimal solutions), to leave the strip and to be packed at other locations. 
These components proved to be quite useful for the effectiveness of the CTS algorithm. We believe that the basic ideas behind these components could be applicable to other optimization problems.

\section{ACKNOWLEDGEMENTS}

We would like to thank the reviewers of the paper. This work was partially supported by three grants from the French "Pays de la Loire" region (MILES, RadaPop, and LigeRO projects). The first author is supported by a Chilean CONICIT scholarship.

\section{REFERENCES}

Alvarez-Valdes, R., Parreño, F., \& Tamarit, J. (2007). A tabu search algorithm for a two-dimensional non-guillotine cutting problem. European Journal of Operational Research, 183, 1167-1182.

Alvarez-Valdes, R., Parreño, F., \& Tamarit, J. (2008). Reactive GRASP for the strip-packing problem. Computers \& Operations Research, 35, 1065-1083.

Araya, I., Neveu, B., \& Riff, M.-C. (2008). An efficient hyperheuristic for strip-packing problems. In C. Cotta, M. Sevaux, \& K. Sörensen (Editors), Adaptive and Multilevel Metaheuristics (volume 136 of Studies in Computational Intelligence, pp. 61-76). Berlin: Springer.

Aşik, O., \& Özcan, E. (2009). Bidirectional best-fit heuristic for orthogonal rectangular strip packing. Annals of Operations Research, 172, 405-427.

Baker, B., Brown, D., \& Katseff, H. (1981). A 5/4 algorithm for two-dimensional packing. Journal of Algorithms, 2, 348-368.

Baker, B., Jr., E. C., \& Rivest, R. (1980). Orthogonal packings in two dimensions. SIAM Journal on Computing, 9, 846-855.

Beasley, J. (2004). A population heuristic for constrained two-dimensional non-guillotine cutting. European Journal of Operational Research, 156, 601-627.

Belov, G., Kartak, V., Rohling, H., \& Scheithauer, G. (2009). One-dimensional relaxations and LP bounds for orthogonal packing. International Transactions in Operational Research, 16, 745-766.

Beltrán Cano, J., Calderón, J., Cabrera, R., Moreno Pérez, J., \& Moreno-Vega, J. (2004). GRASP / VNS hybrid for the strip packing problem. In C. Blum, A. Roli, \& M. Sampels (Editors), First International Workshop on Hybrid Metaheuristics (pp. 79-90).

Błażewicz, J., Moret-Salvador, A., \& Walkowiak, R. (2004). Parallel tabu search approaches for twodimensional cutting. Parallel Processing Letters, 14, 23-32.

Bortfeldt, A. (2006). A genetic algorithm for the two-dimensional strip packing problem with rectangular pieces. European Journal of Operational Research, 172, 814-837.

Burke, E., Kendall, G., \& Whitwell, G. (2009). A simulated annealing enhancement of the best-fit heuristic for the orthogonal stock cutting problem. INFORMS Journal on Computing, 21, 505-516.

Chazelle, B. (1983). The bottom-left bin-packing heuristic: An efficient implementation. IEEE Transactions on Computers, 32, 697-707.

Clautiaux, F., Jouglet, A., Carlier, J., \& Moukrim, A. (2008). A new constraint programming approach for the orthogonal packing problem. Computers \& Operations Research, 35, 944-959.

Coffman Jr., E., Garey, M., Johnson, D., \& Tarjan, R. (1980). Performance bounds for level-oriented two-dimensional packing algorithms. SIAM Journal on Computing, 9, 808-826. 
Dowsland, K., \& Dowsland, W. (1992). Packing problems. European Journal of Operational Research, 56, 2-14.

El Hayek, J., Moukrim, A., \& Negre, S. (2008). New resolution algorithm and pretreatments for the two-dimensional bin-packing problem. Computers \& Operations Research, 35, 3184-3201.

Fowler, R., Paterson, M., \& Tanimoto, S. (1981). Optimal packing and covering in the plane are NPcomplete. Information Processing Letters, 12, 133-137.

Garey, M. R., \& Johnson, D. S. (1979). Computers and Intractability: A Guide to the Theory of NPCompleteness. San Francisco: W. H. Freeman and Company.

Garrido, P., \& Riff, M.-C. (2007). Collaboration between hyperheuristics to solve strip-packing problems. In P. Melin, O. Castillo, L. Aguilar, J. Kacprzyk, \& W. Pedrycz (Editors), Twelfth International Fuzzy Systems Association World Congress (volume 4529 of Lecture Notes in Computer Science, pp. 698-707). Berlin: Springer.

Glover, F. W., \& Laguna M. (1997). Tabu Search. Dordrecht: Kluwer.

Gómez-Villouta, G., Hamiez, J.-P., \& Hao, J.-K. (2008). A dedicated genetic algorithm for twodimensional non-guillotine strip packing. Sixth Mexican International Conference on Artificial Intelligence, Special Session (pp. 264-274). Los Alamitos: IEEE Computer Society.

Gonçalves, J. (2007). A hybrid genetic algorithm-heuristic for a two-dimensional orthogonal packing problem. European Journal of Operational Research, 183, 1212-1229.

Hamiez, J.-P., Robet, J., \& Hao, J.-K. (2009). A tabu search algorithm with direct representation for strip packing. In C. Cotta, \& P. Cowling (Editors), Ninth European Conference on Evolutionary Computation in Combinatorial Optimization (volume 5482 of Lecture Notes in Computer Science, pp. 61-72). Berlin: Springer.

Harren, R., \& van Stee, R. (2009). Improved absolute approximation ratios for two-dimensional packing problems. In I. Dinur, K. Jansen, J. Naor, \& J. Rolim (Editors), Approximation, Randomization, and Combinatorial Optimization (volume 5687 of Lecture Notes in Computer Science, pp. 177-189). Berlin: Springer.

Hopper, E., \& Turton, B. (2001). An empirical investigation of meta-heuristic and heuristic algorithms for a 2D packing problem. European Journal of Operational Research, 128, 34-57.

Ibaraki, T., Imahori, S., \& Yagiura, M. (2008). Hybrid metaheuristics for packing problems. In C. Blum, M. B. Aguilera, A. Roli, \& M. Sampels (Editors), Hybrid Metaheuristics: An Emerging Approach to Optimization (volume 114 of Studies in Computational Intelligence, pp. 185-219). Berlin: Springer.

Imahori, S., Yagiura, M., \& Ibaraki, T. (2003). Local search algorithms for the rectangle packing problem with general spatial costs. Mathematical programming, 97, 543-569.

Imahori, S., Yagiura, M., \& Ibaraki, T. (2005). Improved local search algorithms for the rectangle packing problem with general spatial costs. European Journal of Operational Research, 167, 48-67.

Imahori, S., Yagiura, M., \& Nagamochi, H. (2007). Practical algorithms for two-dimensional packing. In T. Gonzalez (Editor), Handbook of Approximation Algorithms and Metaheuristics (volume 13 of Chapman \& Hall / CRC Computer \& Information Science Series, chapter 36). Boca Raton / London: CRC Press. 
Iori, M., Martello, S., \& Monaci, M. (2003). Metaheuristic algorithms for the strip packing problem. In P. Pardalos, \& V. Korotkikh (Editors), Optimization and Industry: New Frontiers (volume 78 of Applied Optimization, pp. 159-179). Berlin: Springer.

Jansen, K., \& van Stee, R. (2005). On strip packing with rotations. In H. Gabow, \& R. Fagin (Editors), Thirty-Seventh annual ACM symposium on Theory of computing (pp. 755-761). New York: ACM Press.

Kenmochi, M., Imamichi, T., Nonobe, K., Yagiura, M., \& Nagamochi, H. (2009). Exact algorithms for the two-dimensional strip packing problem with and without rotations. European Journal of Operational Research, 198, 73-83.

Lodi, A., Martello, S., \& Monaci, M. (2002). Two-dimensional packing problems: A survey. European Journal of Operational Research, 141, 241-252.

Martello, S., Monaci, M., \& Vigo, D. (2003). An exact approach to the strip packing problem. INFORMS Journal on Computing, 15, 310-319.

Mir, M., \& Imam, M. (2001). A hybrid optimization approach for layout design of unequal-area facilities. Computers \& Industrial Engineering, 39, 49-63.

Neveu, B., Trombettoni, G., \& Araya, I. (2007). Incremental move for strip-packing. In N. Avouris, N. Bourbakis, \& I. Hatzilygeroudis (Editors), Nineteenth IEEE International Conference on Tools with Artificial Intelligence (volume 2, pp. 489-496). Los Alamitos: IEEE Computer Society.

Neveu, B., Trombettoni, G., Araya, I., \& Riff, M.-C. (2008). A strip packing solving method using an incremental move based on maximal holes. International Journal on Artificial Intelligence Tools, 17, 881-901.

Schiermeyer, I. (1994). Reverse-Fit: A 2-optimal algorithm for packing rectangles. In J. van Leeuwen (Editor), Second Annual European Symposium on Algorithms (volume 855 of Lecture Notes in Computer Science, pp. 290-299). Berlin: Springer.

Soh, T., Inoue, K., Tamura, N., Banbara, M., \& Nabeshima, H. (2008). A SAT-based method for solving the two-dimensional strip packing problem. In M. Gavanelli, \& T. Mancini (Editors), Fifteenth International RCRA Workshop on Experimental Evaluation of Algorithms for Solving Problems with Combinatorial Explosion. Aachen: RWTH Aachen University.

Soke, A., \& Bingul, Z. (2006). Hybrid genetic algorithm and simulated annealing for two-dimensional non-guillotine rectangular packing problems. Engineering Applications of Artificial Intelligence, 19, $557-567$.

Terashima-Marín, H., Flores-Álvarez , E., \& Ross, P. (2005). Hyper-heuristics and classifier systems for solving 2D-regular cutting stock problems. 2005 Conference on Genetic and Evolutionary Computation (volume 2, pp. 637-643). New York: ACM Press.

Wäscher, G., Haußner, H., \& Schumann, H. (2007). An improved typology of cutting and packing problems. European Journal of Operational Research, 183, 1109-1130.

Yeung, L., \& Tang,W. (2004). Strip-packing using hybrid genetic approach. Engineering Applications of Artificial Intelligence, 17, 169-177. 